\title{
Normal domain temperature profile in second generation HTS tape wire
}

\author{
Andrey V Malginov ${ }^{1}$, Alexander Yu Kuntsevich ${ }^{1,2}$, Vladimir A Malginov ${ }^{1 *}$ and Leonid S Fleishman ${ }^{3}$
}

\begin{abstract}
Background: Studies of the normal zone in high-temperature superconducting wires are extremely important for power applications, such as fault current limiters, motors, cables etc. We studied the temperature distribution and normal domain propagation in high-temperature superconducting YBCO tape with highly resistive substrate.

Findings: For applied voltages exceeding a certain threshold value the normal domain was found to become unstable and started to propagate along the tape.

Conclusions: The normal domain in superconducting tape with highly resistive substrate appears when voltage is applied to the sample.

At voltages greater than the threshold value, the domain starts to move.

This motion enables us to find the domain temperature and potential profile.
\end{abstract}

Keywords: High-temperature superconductors; Phase transition; Normal zone propagation

\section{Introduction}

Nucleation and propagation of normal zone are of great importance for high-temperature superconductors (HTS) power applications. In previous experimental studies of the normal zone structure in HTS tapes, it was generated e.g. in vacuum by means of an external heater (Daibo et al., 2011, Pelegrin et al., 2011) or by pulsed current (Mader et al., 2011). These conditions are different from those in the HTS power devices where normal zone is generated in liquid $N_{2}$ due to overcritical AC current. In the present paper we generate a normal zone by transport current in the sample. The normal zone appears to be restricted in the finite volume, i.e. we deal with normal domain (ND). Using the time resolved thermocouple and potential probe measurements we can explore the ND spatial structure.

\section{Methods and results}

The sample of YBCO tape wire SuperPower SF12100 was tested in liquid $N_{2}$. The cross-section of the $12 \mathrm{~mm}$ width wire has the following structure: $100 \mu \mathrm{m}$ Hastelloy substrate, $1 \mu \mathrm{m}$ YBCO, $1.5 \mu \mathrm{m} \mathrm{Ag}$. Both nominal and

\footnotetext{
* Correspondence: malginov@sci.lebedev.ru

'Lebedev Physical Institute RAS, 119991, Moscow, Russia

Full list of author information is available at the end of the article
}

measured critical currents of the wire are about $300 \mathrm{~A}$, critical temperature is about $91 \mathrm{~K}$. Figure 1 shows the geometry of the sample with the thermocouple (TC) and potential probes.

The procedure of our AC-measurements is described in detail in Ref. (Fleishman et al., 2010). A constant amplitude $50 \mathrm{~Hz}$ AC voltage $V_{0}$ is applied across the current leads to the HTS sample at the moment $t_{0}$.

Then, current through the sample $I$, temperature of the TC $T$, and voltage in the middle part of the sample $V_{2}$ are being measured with $1 \mathrm{~ms}$ time resolution within $40 \mathrm{~s}$ interval. ND is generated by local overcritical current Joule heating in a specific weak tape segment due to sample inhomogeneity. Location of this weak place $(5 \mathrm{~mm}$ from the right current lead) was determined by eye from liqud nitrogen boiling in the preliminary experiment. TC was soldered near this point.

Figure 2 shows a typical temperature $T$ versus time plot after an application of $V_{0}=0.76 \mathrm{~V}$ AC voltage. The data indicate that the temperature achieves its maximum in $3 \mathrm{~s}$ and almost does not change until switching the voltage off. During this period the voltage $V_{2}$ between the potential probes is approximately zero. 


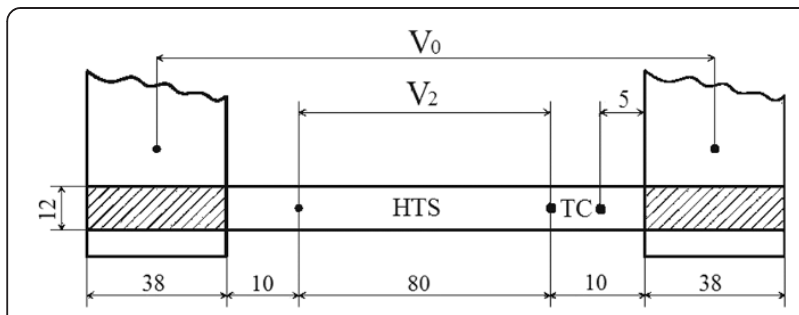

Figure 1 Schematic of the sample with current leads, thermocouple, and probes (distances in $\mathrm{mm}$ ).

The data in the range $0.3 \mathrm{~V}<V_{0}<0.76 \mathrm{~V}$ demonstrate qualitatively similar behavior: once normal domain is formed it stays in the same place and its parameters remain unchanged. It means that for $V_{0}<0.76 \mathrm{~V}$ the normal zone is located between the potential probe and the right current lead, and TC measures the ND temperature.

The temperature versus time dependence changes drastically when the voltage step $V_{0}$ is increased to 0.79 $\mathrm{V}$ (Figure 3). The temperature reaches almost the same maximum value as for lower $V_{0}$ value but instead of being constant decreases afterwards. The rate of temperature drop changes at points $t_{4}^{*}, t_{3}^{*}, t_{2}^{*}, t_{1}^{*}$. At the same time the voltage $V_{2} \neq 0$ appears between the potential probes (Figure 4) exhibiting irregularities at points $t_{1}, t_{2}, t_{3}, t_{4}$. The data in Figures 3 and 4 suggest that ND begins to move as a whole from the sample end towards its center. The irregularities in the $T(t)$ and $V_{2}(t)$ dependences correspond to the motion of specific ND regions across the TC and voltage probe respectively. TC traces the outgoing right edge of the domain. Passing of the left edge is traced by $V_{2}$, e.g. from $t_{1}$ to $t_{2}$ a foremost portion crosses a probe. This portion is cooled by film boiling and has temperature slightly above $77 \mathrm{~K}$. From $t_{2}$ to $t_{3}$

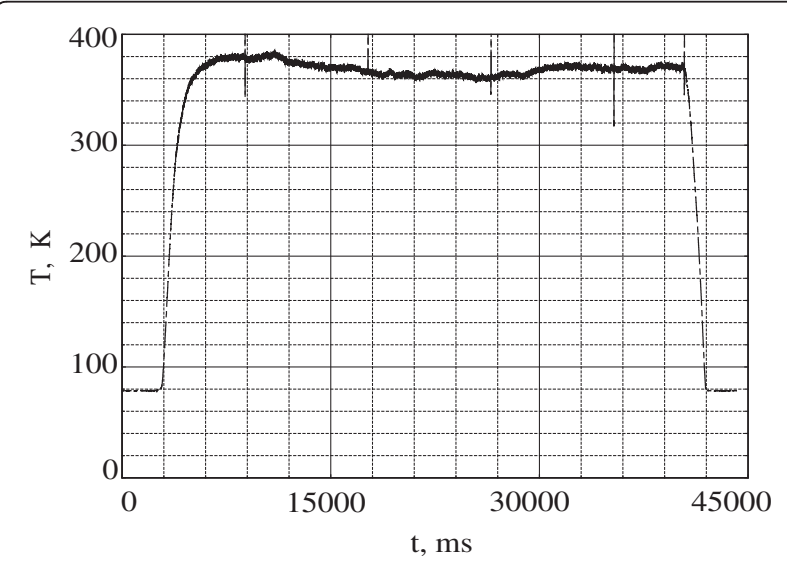

Figure 2 Temperature as a function of time at $V_{0}=0.76 \mathrm{~V}$.

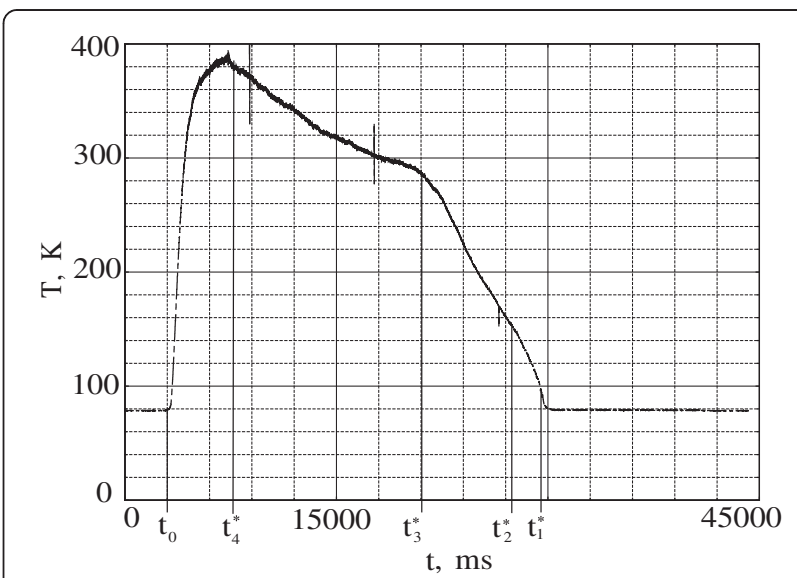

Figure 3 Temperature as a function of time at $V_{0}=0.79 \mathrm{~V}$.

the left edge moves into the segment between the potential probes. From $t_{3}$ to $t_{5}$ the ND top part passes the probe and the temperature is maximal at $t_{4}$. The interval from $t_{4}$ to $t_{5}$ corresponds to the TC measurement interval from $t_{4}^{*}$ to $t_{3}^{*}$. At the period from $t_{3}^{*}$ to $t_{2}^{*}$ the right edge passes TC and after $t_{2}^{*}$ the outermost portion crosses TC position. All the processes occur at the voltage of $V_{0}=0.79 \mathrm{~V}$. Current achieves a stable $I=50 \mathrm{~A}$ level in $3 \mathrm{~s}$ after $t_{0}$. The ND velocity estimated from the rate of the resistance change is about $1 \mathrm{~mm} / \mathrm{s}$. To obtain temperature and potential profiles of the ND as function of distance one should multiply $t$-axis in Figures 3 and 4 by scale factor $1 \mathrm{~mm} / \mathrm{s}$. For higher $V_{0}$ values $\mathrm{ND}$ also drifts towards sample center and both $T(t)$ and $V_{2}(t)$ dependencies demonstrate the similar behavior.

Figure 5 shows the maximum temperature measured by TC as a function of $V_{0}$ value. When $V_{0}<0.3 \mathrm{~V}$ the

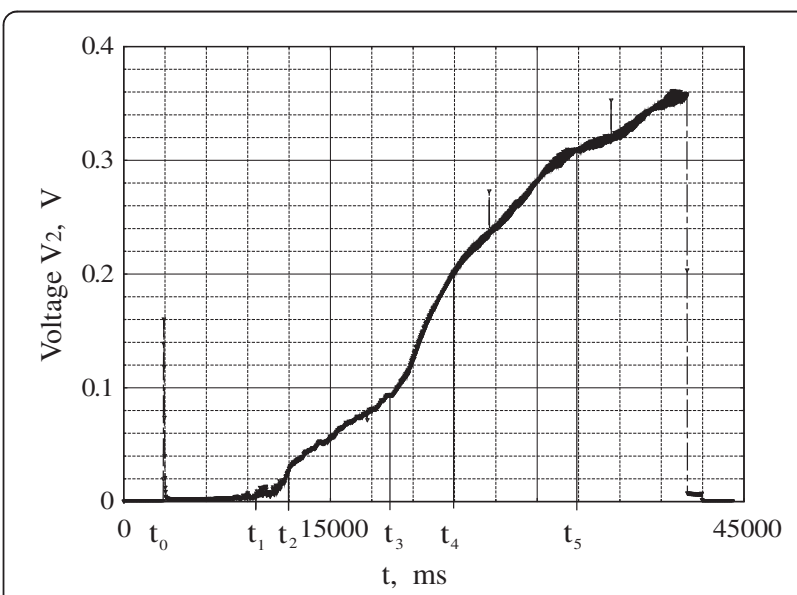

Figure 4 The amplitude value of voltage $V_{2}$ across the middle part of the sample as a function of time for $V_{0}=0.79 \mathrm{~V}$. 


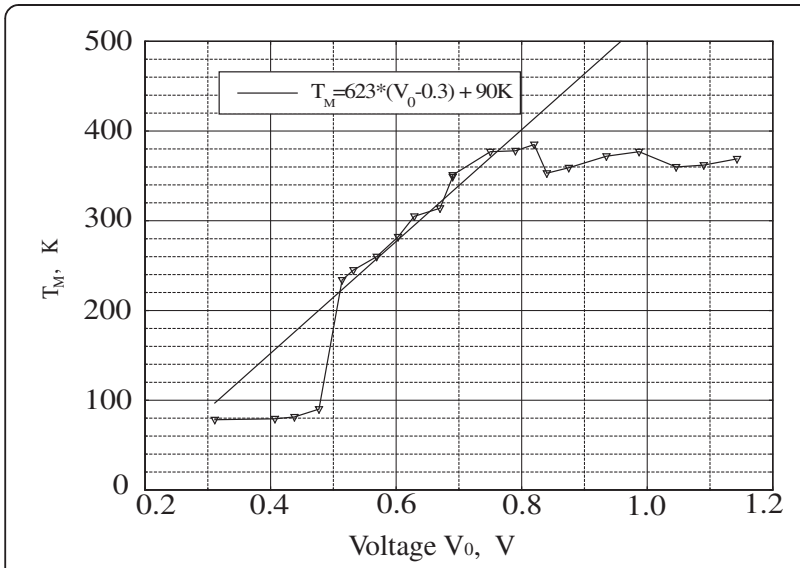

Figure 5 Maximum temperature as a function of amplitude $V_{0}$.
Received: 16 May 2013 Accepted: 18 September 2013

Published: 17 October 2013

\section{References}

Daibo $\mathrm{M}$ et al (2011) Evaluation of the normal-zone propagation characteristics of REBCO coated conductors with laminated Cu tape. IEEE Trans Appl Supercond 21(3):2428-2431

Fleishman LS, Mal'ginov VA, Mal'ginov AV (2010) Ways for increasing the rated capacity of a superconducting current-limiting device. Therm Eng 57 (14):1216-1221

Mader O et al (2011) Investigation of the stability behavior of coated conductors. IEEE Trans Appl Supercond 21(3):3045-3048

Pelegrin $J$ et al (2011) Numerical and experimental analysis of normal zone propagation on 2 G HTS Wires.IEEE Transaction on. Appl Supercond 21 (3):3041-3044

doi:10.1186/2193-1801-2-535

Cite this article as: Malginov et al:: Normal domain temperature profile in second generation HTS tape wire. SpringerPlus 2013 2:535.
ND is so small that TC is out of it and does not respond to heating.

We have shown that for voltages greater than $0.79 \mathrm{~V}$ the domain moves and the TC measures its temperature profile. In the interval $0.3 \mathrm{~V}<V_{0}<0.79 \mathrm{~V}$ the domain stays within the weak place and TC measures the temperature near its top. In this case the ND maximum temperature $T_{\mathrm{M}}$ (in $\mathrm{K}$ ) as a function of applied voltage $V_{0}$ (in $\mathrm{V}$ ) is approximated by: $T_{\mathrm{M}}=623\left(V_{0}-0.3\right)+90$. This fit allows one to estimate the upper boundary of the voltage $(V \sim 1 \mathrm{~V})$, for which the tape survives $(T \sim$ $500 \mathrm{~K})$.

\section{Conclusions}

To summarize, the above experimental data indicate that the normal domain in superconducting tape with highly resistive substrate appears when voltage is applied to the sample. At voltages greater than $0.79 \mathrm{~V}$ the domain starts to move. This motion enables us to find the domain temperature and potential profile from the simultaneous measurements with a thermocouple and potential probes.

\section{Competing interests}

The authors declare that they have no competing interests.

\section{Authors' contributions}

All authors planned and designed the experiment, read and approved the final manuscript.

\section{Acknowledgements}

This work was supported by Russian Foundation for Basic Research (Grant 14-08-00418-a and Grant 12-08-31415-mol-a), Programs of Russian Academy of Sciences, Russian Ministry of Education and Sciences (Grant 8203), Russian science support foundation and using facilities of the Shared Research Equipment Center at LPI.

\section{Author details}

'Lebedev Physical Institute RAS, 119991, Moscow, Russia. ${ }^{2}$ Moscow Institute of Physics and Technology (State University), 141700, Dolgoprudny, Russia. ${ }^{3}$ Krzhizhanovsky Power Engineering Institute, 119991, Moscow, Russia.

\section{Submit your manuscript to a SpringerOpen ${ }^{\circ}$ journal and benefit from:}

- Convenient online submission

Rigorous peer review

- Immediate publication on acceptance

- Open access: articles freely available online

- High visibility within the field

- Retaining the copyright to your article

Submit your next manuscript at springeropen.com 